\title{
Demographic, Clinical and Polysomnographic Characteristics of Childhood- and Adult-Onset Sleepwalking in Adults
}

\author{
Panagiotis Bargiotas $^{a} \quad$ Iris Arnet $^{a} \quad$ Michael Frei $^{a} \quad$ Christian R. Baumann ${ }^{b}$ \\ Kaspar Schindler ${ }^{a}$ Claudio L. Bassetti ${ }^{a}$

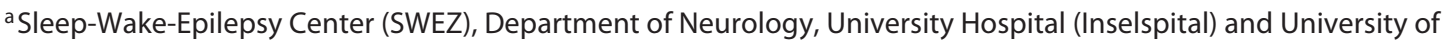 \\ Bern, Bern, Switzerland; bepartment of Neurology, University Hospital Zurich, Zurich, Switzerland
}

\section{Keywords}

Sleep disorders · Adult sleepwalking · Somnambulism ·

Parasomnia $\cdot$ Polysomnography $\cdot$ Violent behavior

\begin{abstract}
Background: Sleepwalking (SW) is found to affect children predominantly, but it can persist or appear de novo even among adults. In this study, we assessed the demographic, clinical and polysomnographic profile, trigger factors and associated comorbidities of adult-onset (AO-SW) and childhood-onset (CO-SW) adult sleepwalkers. Methods: In adult sleepwalkers, a structured clinical interview, a battery of questionnaires, video-polysomnography (v-PSG) and standard electroencephalography (EEG) were performed. $\boldsymbol{R} \boldsymbol{e}$ sults: Among 63 sleepwalkers, $45 \%$ had $\geq 1$ episodes/month, $54 \%$ had partial recall of the episodes and $36 \%$ reported trigger factors for SW. Almost all subjects reported co-occurring parasomnias. In v-PSG, 4\% exhibited episodes of SW, 17\% confusional arousals, $21 \%$ had an increased apnea-hypopnea-index and $6 \%$ exhibited features of an overlap parasomnia disorder. In our cohort, 73\% reported CO-SW and 27\% AO-SW. In subjects with AO-SW, positive family history for parasomnias was found in 33\% (vs. $49 \%$ in CO-SW), neuro-
\end{abstract}

\section{KARGER}

(c) 2017 S. Karger AG, Basel

E-Mail karger@karger.com

www.karger.com/ene logical comorbidities in $44 \%$ (vs. 14\%), psychiatric comorbidities in $25 \%$ (vs. 33\%), EEG abnormalities in 50\% (vs. 29\%). Violence during SW episodes was more frequent in males and in subjects with CO-SW (45\% for self-injury and $44 \%$ for violent behaviour vs. 33 and $29 \%$ respectively in the AO-SW group). Conclusions: Adult SW represents a complex and potentially dangerous condition. The characteristics of AOSW often differ from those of CO-SW.

(C) 2017 S. Karger AG, Basel

\section{Introduction}

Sleepwalking (SW) is considered to be a disorder of impaired arousal [1]. This sleep disorder is a non-rapid eye movement sleep parasomnia, which occurs predominantly out of slow wave sleep and is characterized by a state of dissociated consciousness, in which phenomena of the sleeping and waking states coexist $[1,2]$. SW is commonly found in the general population and the lifetime prevalence is about $29 \%$. It typically affects children reaching a peak at around 10-13 years [3], disappearing in about $75 \%$ of the affected during the adolescence $[4,5]$. SW can also persist or appear de novo in adulthood.

Dr. med. Panagiotis Bargiotas

Department of Neurology, Inselspital, Bern University Hospital Freiburgstrasse 18 CH-3010 Bern (Switzerland)

E-Mail panagiotis.bargiotas@insel.ch 
Among adult sleepwalkers, approximately 13\% develop this sleep disturbance during adulthood [5]. The overall prevalence of SW in adulthood is about 4\% [6].

Only a few studies in the past have assessed characteristics and comorbidities of SW. In the study by Lopez et al. [7], SW was associated with an altered quality of life and several symptoms including daytime sleepiness, fatigue, insomnia, depression and anxiety. In contrast, Labelle et al. [8] reported that there was no association between adult SW and clinically significant levels of depression or anxiety and even if present, these symptoms had little impact on the phenomenological features of SW. Commonly reported trigger factors for SW include sleep deprivation and stress [9]. In addition, subjects with a longer history of SW reported more frequent episodes of SW [9]. Lam et al. [10] showed a higher prevalence of AOSW in a psychiatric outpatient cohort compared to the general population.

Data regarding differences between childhood-SW (CO-SW) and adult-onset SW (AO-SW) is limited. Lopez et al. [7] reported that subjects with late-onset (subjects older than 9 years) primary SW (subjects with comorbidities were excluded) had lower incidence of violent behaviours and were more prone to worsening of their SW over time compared to those with early-onset SW [7]. However, in another cohort, Bušková et al. [9] found no association between age at onset of SW with violent and injurious behaviour.

Most of the above-mentioned studies focused only on specific aspects of SW profiles (e.g., psychiatric comorbidities), reported results independent of the age at onset of SW (childhood- vs. adulthood-onset) or excluded secondary SW related to comorbidities. The current study aimed to describe demographic data, clinical and polysomnographic features, trigger factors and associated comorbidities of AO-SW and CO-SW adult sleepwalkers.

\section{Patients and Methods}

Data obtained as part of the clinical routine from 78 subjects who were referred, over a period of 12 years, to the sleep centres of the University Hospital Bern and the University Hospital Zürich and reported SW was retrospectively analyzed. The adult subjects were classified as CO-SW if the first episode of SW occurred during childhood ( $<18$ years) and as AO-SW if it occurred during adulthood.

Fifteen subjects were excluded from analysis due to the high amount of missing data (including demographic, clinical data and objective assessments) and therefore, 63 subjects were included in the analysis. A standard video-polysomnography (v-PSG) has been performed in 52 of them as previously described [11]. The v-
PSG consisted of 6 channel electroencephalography (EEG; F3/A2, F4/A1, C3/A2, C4/A1, O1/A2, O2/A1), left and right electrooculography, submental, left and right anterior tibialis, flexor carpi radialis and adductor digiti minimi electromyography, electrocardiography, respiratory flow and effort, and pulse-oximetry. Sleep stages and sleep-associated events were manually scored according to the American Academy of Sleep Medicine scoring manual [12]. In 43 subjects, a 10/20 routine EEG was also performed.

The clinical and epidemiological sleep-wake profile of the patients was assessed by a structured clinical interview based on the "Bern Sleep Questionnaire". This questionnaire contains questions regarding demographics, reasons for referral to sleep laboratory, general information about sleep-wake habits and sleep problems, breathing and circulation, parasomnias and potential trigger factors, dreaming, dream enactment behaviour, waking-up, daytime tiredness and sleepiness, cataplexy, hallucinations, stress, well-being, neurological, psychiatric and other comorbidities, family history and questions regarding pregnancy in female participants. In addition, patient's medical records were reviewed to obtain additional information regarding reasons for referral to sleep laboratory, neurological and psychiatric comorbidities and medication. The time that would be needed to complete the entire questionnaire was around $90 \mathrm{~min}$.

Epworth sleepiness scale (ESS) [13] was also performed and excessive daytime sleepiness was defined as an ESS score $\geq 10$ [14].

The study was approved by the institutional review board of the departments and patients had given their written informed consent for using their data for scientific purposes.

\section{Statistics}

Univariate and bivariate analyses: depending on categorical or continuous variable and the distribution of the data, chi-square test/Fisher's exact test and unpaired Student $t$ test were performed. The SD was used in conjunction with the mean to summarize continuous data. The level of significance was 0.05 in all tests. All tests were calculated using statistical package for social sciences, version 18 (SPSS-18).

\section{Results}

Epidemiological, Clinical and Polysomnographic

Profile of Sleepwalkers

Data from 63 adult sleepwalkers was collected (age $39.1 \pm 15.3$, range $18-80$ years, 41 male subjects, 46 subjects with CO-SW, 17 subjects with AO-SW). Excessive daytime sleepiness (ESS $\geq 10$ ) was present in $60 \%$ of the subjects. Mean ESS was $10.2 \pm 5.2$. Among the subjects, $14 \%$ reported alcohol consumption on a regular basis. Demographic characteristics in our cohort are presented in Table 1.

In almost half of the cases, SW was not the reason for being referred to the sleep centre. The most frequent reasons for referral are presented in Table 2. Most sleepwalkers in our cohort (45\%) had $\geq 1$ episode/month, $22 \% \leq 1$ episode/ month, $29 \%$ had weekly episodes and only $4 \%$ reported very 
Table 1. Demographic characteristics of adult sleepwalkers

\begin{tabular}{ll}
\hline Demographics & Mean \pm SD \\
\hline Age, years & $39.1 \pm 15.3$ \\
$\quad$ Males & $39.8 \pm 16.1$ \\
Females & $38.2 \pm 14.4$ \\
Males, \% & 65.1 \\
BMI & $26.0 \pm 5.3$ \\
$\quad$ Males & $25.8 \pm 5.3$ \\
Females & $26.2 \pm 5.1$ \\
ESS & $10.2 \pm 5.2$ \\
$\quad$ Males & $10.3 \pm 4.0$ \\
Females & $11.1 \pm 7.3$ \\
\hline
\end{tabular}

BMI, body mass index; ESS, Epworth Sleepiness Scale.

Table 2. Reasons for referral to the sleep laboratory

\begin{tabular}{lc}
\hline Referral & Percentage, \% \\
\hline Suspected sleepwalking & 60 \\
Suspected sleep apnea & 12 \\
Insomnia or hypersomnia & 9 \\
Suspected epilepsy & 4 \\
Other reasons & 15 \\
\hline
\end{tabular}

Table 3. Polysomnographic features of subjects with adult sleepwalking

\begin{tabular}{|c|c|}
\hline PSG variables & Mean \pm SD \\
\hline AHI, $n / \mathrm{h}$ & $8.2 \pm 13.1$ \\
\hline Mean desaturation, \% & $4.3 \pm 8.9$ \\
\hline Sleep latency, $\min$ & $20.2 \pm 44.0$ \\
\hline REM latency, min & $125.2 \pm 79.5$ \\
\hline Sleep efficiency ${ }^{\mathrm{a}}$ & $81.1 \pm 17.3$ \\
\hline REM sleep, $\%^{a}$ & $10.1 \pm 6.2$ \\
\hline NREM1 sleep, $\%^{\mathrm{a}}$ & $22.4 \pm 11.8$ \\
\hline NREM2 sleep, $\%^{\mathrm{a}}$ & $41.1 \pm 12.2$ \\
\hline NREM3 sleep, $\%^{\mathrm{a}}$ & $14.3 \pm 7.1$ \\
\hline PLM-index & $8.1 \pm 28.8$ \\
\hline
\end{tabular}

${ }^{\text {a }}$ Percentage of the total sleep time, $n=52$

PSG, polysomnography; AHI, Apnoe-Hypopnea Index; REM, rapid eye movement; NREM, non-rapid eye movement; PLM, Periodic limb movements.

frequent episodes $\geq 2$ /week. More than half of the patients (54\%) had partial recall of the episodes and $36 \%$ reported at least one trigger factor for SW. The most frequent trigger factors were stress (68\%), full moon (25\%), anxiety (21\%), alcohol consumption and sleep deprivation (18\%).

Characteristics of Adult SW
Almost all sleepwalkers (98\%) reported co-occurring parasomnias. Sleep talking (91\%), night terrors (69\%), bruxismus (54\%) and nocturnal eating behaviour (50\%) were the most frequently occurring events. In addition, hallucinations (45\%), sleep paralysis (35\%) and enuresis (7\%) were frequently reported.

Among 52 sleepwalkers who underwent a v-PSG, 27 subjects (52\%) had a sleep latency less than $10 \mathrm{~min}$ and 7 subjects a REM latency less than $60 \mathrm{~min}, 24$ subjects (46\%) had a sleep efficiency below $85 \%, 11$ subjects (21\%) had an apnea-hypopnea-index higher than $10 / \mathrm{h}$, and 11 subjects $(21 \%)$ had a PLM (periodic limb movements)-index higher than 15/h. Sleep architecture was often characterized by an increased percentage of stage 1 sleep and decreased rapid eye movement (REM) sleep and slow wave sleep compared to elsewhere reported age-dependent normal values [15]. Table 3 presents the main polysomnographic features of subjects with adult SW.

During v-PSG, episodes of SW were observed in 2 subjects (4\%) and confusional arousals in 9 subjects (17\%). Notably, in 3 patients (6\%), the criteria for the co-existence of a REM-parasomnia (increased phasic muscle activity, jerks and increased muscle tone or partial loss of REM atonia combined with signs of dream enactment) were fulfilled [16].

\section{Childhood- and Adult-Onset Sleepwalking}

Among 63 sleepwalkers, 46 subjects reported CO-SW and 17 subjects reported AO-SW. Our cohort included predominantly males (65\%); however, regarding the age at onset of SW no male-female differences were found. Patients with CO-SW reported more often than those in the AO-SW group a positive family history for parasomnias ( 49 vs. $33 \% ; p=0.04$ ). Neurological comorbidities were significantly more often in the AO-SW than in the CO-SW (44 vs. $14 \% ; p=0.02$ ). The most frequent were traumatic brain injury, parkinsonism, stroke, alcohol-related encephalopathy and perinatal cerebral dysfunction. In the AO-SW group, there was a trend for more frequent occurrences of EEG abnormalities compared to the COSW group; however, the difference was not significant (50 vs. $29 \%$; $p=0.24)$. Typical EEG abnormalities included slowing activity in basic rhythm and theta activity. No epileptic activity was reported.

In our cohort, $29 \%$ of the subjects had a psychiatric comorbidity, the most frequent one being depression (23\%). In addition, $14 \%$ reported a history of alcohol abuse. No differences were observed in the frequency of psychiatric comorbidities or alcohol abuse between the 2 groups ( $25 \%$ in $\mathrm{AO}-\mathrm{SW}$ vs. $33 \%$ in CO-SW; $p=0.39$ ). 
Table 4. Differences between AO- and CO-SW

\begin{tabular}{llll}
\hline & CO-SW, \% & AO-SW, \% & $p$ value \\
\hline Males & 61 & 71 & 0.34 \\
Positive family history for & & & \\
$\quad$ parasomnias & 49 & 33 & 0.04 \\
$\quad$ Neurological comorbidities & 14 & 44 & 0.02 \\
Psychiatric comorbidities & 33 & 25 & 0.39 \\
$\begin{array}{l}\text { Abnormalities in EEG } \\
\text { Sleepwalking-related }\end{array} \quad 29$ & 50 & 0.24 \\
$\quad$ self-injuries & 45 & 33 & 0.32 \\
$\quad$ Violent behavior during & & & \\
$\quad$ sleepwalking & 44 & 29 & 0.03 \\
\hline
\end{tabular}

AO-SW, adult-onset sleepwalkers; CO-SW, childhood-onset sleepwalkers; EEG, electroencephalography.

In our cohort, $40 \%$ of the adult sleepwalkers reported violent behaviour during SW and $42 \%$ reported self-injuries. Among them 72 (62.5\%) were males. In the AO-SW group, $45 \%$ of the patients reported episodes with selfinjury and $44 \%$ episodes with violent behaviour. For the patients in the CO-SW group, these percentages were $33 \%(p=0.32)$ and $29 \%(p=0.03)$ for self-injury and violent behaviour respectively. The differences between $\mathrm{CO}-$ and AO-SW are presented in Table 4.

\section{Discussion}

In the current study, we assessed characteristics of adult sleepwalkers and differences between AO- and CO-SW.

The profile of subjects with AO-SW differs from that of those with CO-SW. We confirmed previous reports that nearly $40 \%$ of individuals with SW had a family history of such a disorder [6]. However, in the subgroup analysis, not surprisingly, familial occurrence was significantly more frequent in the CO-SW compared to the AO-SW group. Neurological comorbidities were present in $23 \%$ of the subjects and were twice more frequent in the AO-SW group compared to subjects with CO-SW. Consequently, more subjects in the AO-SW group exhibited EEG abnormalities. In our cohort, nearly one-third of the subjects reported a psychiatric comorbidity; however, we could not confirm previous reports that psychiatric comorbidities or alcohol abuse are more often in subjects with $\mathrm{AO}$ SW. We confirmed previous reports that violent episodes during SW are more frequently reported by male sleepwalkers [17] which might, at least partially, explain the more frequent referral of male sleepwalkers to our sleep centres. Adults with CO-SW reported less self-injuries, and the episodes of SW were significantly less violent compared to those in adults with AO-SW.

Often the first therapeutic approach of SW is based on the treatment of comorbidities or the management of trigger factors. Therefore, we focused on the demographic, clinical and polysomnographic profile, trigger factors and associated comorbidities of adult sleepwalkers. The demographic characteristics of our cohort did not differ significantly from those reported from previous studies. Sleep walking was more frequently reported in the category $\geq 1$ episode/month and less frequent in the category $\geq 2$ episodes/week. Consistent with previous reports [7], one-third of the subjects reported at least one SW trigger factor. Stress was mentioned by almost two-thirds of them and sleep deprivation, anxiety and alcohol consumption were also frequently reported. Interestingly, full moon was reported by $25 \%$ of our patients as a trigger factor, suggesting a periodic occurrence of the episodes, whereas in previous studies, it was rarely mentioned [9] or not mentioned at all. In our cohort, as in previous studies [18, 19], there were more males among sleepwalkers. This does not necessarily reflect an association of SW with gender [6] but possibly differences in the referral rates, which are likely due to the fact that male sleepwalkers have more frequent violent episodes than female sleepwalkers.

During night polysomnography, sleep efficiency and frequency of confusional arousals or episodes of SW in our cohort were comparable to previous studies [9]. However, sleepiness was almost 4-times and sleep disordered breathing (SDB) almost twice more frequent than previously reported [9]. It still remains unclear if and why subjects with SDB become more susceptible to SW. Based on the hypothesis that sleep disruption due to SDB could increase arousability, which under specific conditions (e.g., trigger factors) could lead to SW [19], early studies suggested that obstructive sleep apnea syndrome is associated with SW and treatment of the SDB could reduce susceptibility to SW [19-21].

Comorbid parasomnias are very frequent among sleepwalkers. The frequency of comorbid sleep talking and night terrors in our cohort was similar to previous reports [7]. To our knowledge, this study is the first to report the prevalence of bruxism, nocturnal hallucinations, sleep paralysis and enuresis in a cohort of sleepwalkers. Interestingly, $6 \%$ of the patients also fulfilled the polysomnographic and clinical criteria of a REM parasomnia, suggesting the existence of an overlap parasomnia. The prevalence of REM sleep behaviour disorder (RBD) is about $0.5 \%$ in the general population and $2 \%$ in 
the older adult population [22]. Our data supports previous reports of an increased prevalence of SW among patients with RBD $[11,23]$. RBD is considered a preclinical marker for neurodegenerative disorders and therefore the prevalence of overlap parasomnia among sleepwalkers needs further investigation.

The comparison of unequal sample sizes is a limitation of the current study. However, considering the low percentage of AO-SW among adult sleepwalkers (e.g., 13\% in Lopez et al. [7], 20\% in Labelle et al. [8]), the formation of equal sample sizes would require a prospective approach.

Our data indicates that adult SW represents a complex and potentially dangerous condition and underpin the importance of a detailed history taking, clinical, and polysomnographic assessment of adult sleepwalkers. Psychiatric and neurological comorbidities (for the latest espe- cially in subjects with AO-SW) but also comorbid sleeprelated disorders (e.g., SDB, overlap parasomnia) should be assessed and if indicated should be treated. This, together with a comprehensive counseling regarding prognosis (e.g., increased risk of experiencing violent or even self-injurious behaviour especially for subjects with $\mathrm{CO}$ SW) consists an essential initial step towards the successful management of SW.

\section{Disclosure Statement}

P.B. has received an "Inselgrant" from Inselspital, Bern. He has also received congress fees and travel reimbursements from Lundbeck Foundation. I.A., M.F., K.S., and C.L.B. report no financial disclosures. C.R.B. received grants from Swiss National Science Foundation, HSM-2 Grant Canton of Zurich, UCB Pharma, AbbVie Pharma.

\section{References}

1 Broughton RJ: Sleep disorders: disorders of arousal? Enuresis, somnambulism, and nightmares occur in confusional states of arousal, not in "dreaming sleep". Science 1968;159: 1070-1078.

2 Kales A, Jacobson A, Paulson MJ, Kales JD, Walter RD: Somnambulism: psychophysiological correlates. I. All-night EEG studies. Arch Gen Psychiatry 1966;14:586-594.

3 Hublin C, Kaprio J: Genetic aspects and genetic epidemiology of parasomnias. Sleep Med Rev 2003;7:413-421.

4 Kales A, Soldatos CR, Caldwell AB, et al: Somnambulism. Clinical characteristics and personality patterns. Arch Gen Psychiatry 1980; 37:1406-1410.

5 Hublin C, Kaprio J, Partinen M, Heikkilä K, Koskenvuo M: Prevalence and genetics of sleepwalking: a population-based twin study. Neurology 1997;48:177-181.

6 Ohayon MM, Mahowald MW, Dauvilliers Y, Krystal AD, Leger D: Prevalence and comorbidity of nocturnal wandering in the U.S. adult general population. Neurology 2012;78: 1583-1589.

7 Lopez R, Jaussent I, Scholz S, Bayard S, Montplaisir J, Dauvilliers Y: Functional impairment in adult sleepwalkers: a case-control study. Sleep 2013;36:345-351.

8 Labelle MA, Desautels A, Montplaisir J, Zadra A: Psychopathologic correlates of adult sleepwalking. Sleep Med 2013;14:1348-1355.
9 Bušková J, Piško J, Pastorek L, Šonka K: The course and character of sleepwalking in adulthood: a clinical and polysomnographic study. Behav Sleep Med 2015;13:169177.

10 Lam SP, Fong SY, Yu MW, Li SX, Wing YK: Sleepwalking in psychiatric patients: comparison of childhood and adult onset. Aust N Z J Psychiatry 2009;43:426-430.

11 Di Fabio N, Poryazova R, Oberholzer M, Baumann CR, Bassetti CL: Sleepwalking, REM sleep behaviour disorder and overlap parasomnia in patients with Parkinson's disease. Eur Neurol 2013;70:297-303.

12 AASM Manual for the Scoring of Sleep and Associated Events: Rules TaTS, ed 1. Westchester/IL, American Academy of Sleep Medicine, 2007.

13 Johns MW: A new method for measuring daytime sleepiness: the Epworth sleepiness scale. Sleep 1991;14:540-545.

14 Bloch KE, Schoch OD, Zhang JN, Russi EW: German version of the Epworth Sleepiness Scale. Respiration 1999;66:440-447.

15 Ohayon MM, Carskadon MA, Guilleminault C, Vitiello MV: Meta-analysis of quantitative sleep parameters from childhood to old age in healthy individuals: developing normative sleep values across the human lifespan. Sleep 2004;27:1255-1273.

16 Frauscher B, Iranzo A, Gaig C, et al: Normative EMG values during REM sleep for the di- agnosis of REM sleep behavior disorder. Sleep 2012;35:835-847.

17 Siclari F, Khatami R, Urbaniok F, et al: Violence in sleep. Brain2010;133:3494-3509.

18 Schenck CH, Boyd JL, Mahowald MW: A parasomnia overlap disorder involving sleepwalking, sleep terrors, and REM sleep behavior disorder in 33 polysomnographically confirmed cases. Sleep 1997;20:972-981.

19 Guilleminault C, Kirisoglu C, Bao G, Arias V, Chan A, Li KK: Adult chronic sleepwalking and its treatment based on polysomnography. Brain 2005;128:1062-1069.

20 Ohayon MM, Guilleminault C, Priest RG: Night terrors, sleepwalking, and confusional arousals in the general population: their frequency and relationship to other sleep and mental disorders. J Clin Psychiatry 1999;60: 268-276; quiz 277.

21 Espa F, Dauvilliers Y, Ondze B, Billiard M, Besset A: Arousal reactions in sleepwalking and night terrors in adults: the role of respiratory events. Sleep 2002;25:871-875.

22 Kang SH, Yoon IY, Lee SD, Han JW, Kim TH, Kim KW: REM sleep behavior disorder in the Korean elderly population: prevalence and clinical characteristics. Sleep 2013;36:11471152.

23 Lin FC, Lai CL, Huang P, Liu CK, Hsu CY: The rapid-eye-movement sleep behavior disorder in Chinese-Taiwanese patients. Psychiatry Clin Neurosci 2009;63:557-562. 